\title{
Transition between the discharge regimes of high power impulse magnetron sputtering and conventional direct current magnetron sputtering
}

\author{
Daniel Lundin, Nils Brenning, Daniel Jädernäs, Petter Larsson, Erik Wallin, \\ Martina Lattemann, Michael A Raadu and Ulf Helmersson
}

\section{Linköping University Post Print}

N.B.: When citing this work, cite the original article.

Original Publication:

Daniel Lundin, Nils Brenning, Daniel Jädernäs, Petter Larsson, Erik Wallin, Martina Lattemann, Michael A Raadu and Ulf Helmersson, Transition between the discharge regimes of high power impulse magnetron sputtering and conventional direct current magnetron sputtering, 2009, PLASMA SOURCES SCIENCE and TECHNOLOGY, (18), 4, 045008.

http://dx.doi.org/10.1088/0963-0252/18/4/045008

Copyright: Institute of Physics

http://www.iop.org/

Postprint available at: Linköping University Electronic Press

http://urn.kb.se/resolve?urn=urn:nbn:se:liu:diva-51382 


\title{
Transition between the discharge regimes of high power impulse magnetron sputtering and conventional direct current magnetron sputtering
}

\author{
Daniel Lundin ${ }^{1}$, Nils Brenning ${ }^{2}$, Daniel Jädernäs ${ }^{1}$, Petter Larsson ${ }^{1}$, Erik Wallin ${ }^{1}$, \\ Martina Lattemann ${ }^{3,4}$, Michael A. Raadu ${ }^{2}$ and Ulf Helmersson ${ }^{1}$
}

1 Plasma \& Coatings Physics Division, IFM-Materials Physics, Linköping University, SE-581 83 Linköping, Sweden

${ }^{2}$ Division of Space and Plasma Physics, School of Electrical Engineering, Royal Institute of Technology, SE-100 44 Stockholm, Sweden

3 Joint Research Laboratory Nanomaterials, Technische Universität Darmstadt and Forschungszentrum Karlsruhe GmbH, 64287 Darmstadt, Germany

${ }^{4}$ Institute for Nanotechnology, Forschungszentrum Karlsruhe GmbH, Postfach 3640, 76021 Karlsruhe, Germany

E-mail: danlu@ifm.liu.se

\begin{abstract}
Current and voltage have been measured in a pulsed high power impulse magnetron sputtering (HiPIMS) system for discharge pulses longer than $100 \mu$ s. Two different current regimes could clearly be distinguished during the pulses: (1) A high-current transient followed by (2) a plateau at lower current. These results provide a link between the HiPIMS and the direct current magnetron sputtering (DCMS) discharge regimes. At high applied negative voltages the high-current transient had the characteristics of HiPIMS pulses, while at lower voltages the plateau values agreed with currents in DCMS using the same applied voltage. The current behavior was found to be strongly correlated with the chamber gas pressure, where increasing gas pressure resulted in increasing peak current and plateau current. Based on these experiments it is here suggested that the high-current transients cause a depletion of the working gas in the area in front of the target, and thereby a transition to a DCMS-like high voltage, lower current regime.
\end{abstract}


D. Lundin et al. Transition from a highly ionized plasma to a low density plasma in a high power impulse magnetron discharge

\section{Introduction}

The method of high power impulse magnetron sputtering (HiPIMS) [1, 2], has become a wellestablished coating technology within the scientific community and the industrial interest is growing rapidly. It is characterized by a very high plasma density, on the order of $10^{19} \mathrm{~m}^{-3}$, about three orders of magnitude higher compared to conventional direct current magnetron sputtering (DCMS) $[3,4]$. This leads to a high ionization rate of the sputtered material. A large fraction of the sputtered material being ionized leads to a better control of the film growth through the use of substrate bias that can be used to accelerate and guide the deposition material. HiPIMS is therefore a promising technique for coatings on complex surfaces, such as filling of trenches in integrated circuits [2]. It has also been shown that the use of HiPIMS has resulted in improved film quality $[5,6]$, particularly at low substrate temperatures.

Previous publications on HiPIMS discharges have focused on the high power pulse as observed for short pulse-on times [7]. Alami and co-workers investigated the U-I characteristics for different voltages identifying different operation modes [8]. It was concluded that the different modes were caused by different plasma confinement strengths and gas depletion, as verified by a decrease of the optical emission spectroscopy intensity measured for $\mathrm{Ar}$ at high discharge voltages. For very long pulses the process must approach the steady-state conditions achieved during DCMS and is the subject of the present study.

In this article it is shown that the usual short $(<100 \mu \mathrm{s})$ HiPIMS pulses fall in a transient high-current state, obtained when initiating a magnetron plasma. Such high-current transients, with corresponding high plasma densities, do not necessarily require high voltages. Even when using voltages comparably to DCMS discharges, a higher current and higher plasma density are achieved initially. The transition from the high-current transient to the steady-state regime is 
D. Lundin et al. Transition from a highly ionized plasma to a low density plasma in a high power impulse magnetron discharge

proposed to be dominated by gas rarefaction, but also other possible mechanisms affecting this transition phase are discussed.

\section{Experimental details}

The deposition system consisted of a cylindrical vacuum chamber pumped with a turbo-molecular pump to a background pressure of about $1.3 \times 10^{-5} \mathrm{~Pa}$. The system was equipped with a standard planar circular magnetron, $0.076 \mathrm{~m}$ in diameter, mounted with a $\mathrm{Cr}(99.9 \%)$ target. $\mathrm{Ar}$ (with a minimum purity of $99.9997 \%$ ) was used as the sputtering gas. In this work, high-voltage pulses were applied between the cathode (target) and the chamber walls using a pulsing unit (SPIK 1000A, Melec) fed by a direct current (DC) power supply (MDX Pinnacle, Advanced Energy) delivering constant voltage pulses of controlled length, and capable of sustaining high instantaneous currents over a long period of time (several hundred $\mu$ s). Thereby, the current behavior for different applied negative target voltages and Ar pressures could be investigated as function of pulse-on time. For conventional DC reference measurements a DC power supply was connected to the magnetron, without the pulsing unit, and set to the same voltage as for the HiPIMS case.

The target voltage and target current were monitored and recorded on a Tektronix TDS 520C oscilloscope. A Tektronix P6015 high voltage probe measured the target voltage. The current was measured with a Tektronix CT-04 high current transformer together with a Tektronix TCP202 current probe.

\section{Experimental results}

The obtained discharge current characteristics, $I_{D}(t)$, for different pulse-on times $\left(t_{o n}=100-\right.$ $500 \mu \mathrm{s})$ are shown in Figure 1. The pulse-off time was in all cases $9.9 \mathrm{~ms}$. For all pulse-on times a high-current peak, typical for HiPIMS, was measured. However, as the pulse-on time was increased above $100 \mu \mathrm{s}$, a current plateau developed at about $I_{p l}=3.5 \mathrm{~A}$. The plateau was stable 
D. Lundin et al. Transition from a highly ionized plasma to a low density plasma in a high power impulse magnetron discharge

throughout the remainder of the pulse and was cut off at the end of the pulse. The negative voltage pulse was fixed at $U=700 \mathrm{~V}$. For the longer pulses ( $t_{o n} \geq 200 \mu \mathrm{s}$ ) the current can be described by three distinct regions as indicated in the figure: (I) the plasma ignition, which is followed by (II), a decay after passing the current maximum, and finally going into a steady state regime in (III). Another feature that can be seen is a small negative current during pulse-off. This is due to the different escape paths taken by electrons and ions when the pulse is turned off: the magnetized electrons travel along the magnetic field lines towards the cathode, while the ions are lost to all surfaces. This gives rise to a reversed current peak. Note that the high-current transient before the plateau phase is not a power supply artifact; the voltage level is kept almost constant throughout the whole pulse as seen in Figure 2. If the decay in current would have been caused by a too small bank of capacitors, the measured applied voltage would also have decreased, which however was not observed.

The peak value during the high-current transient, the plateau, and the DCMS discharge current are plotted as functions of the discharge voltage in Figure 3. For applied negative voltages less than $580 \mathrm{~V}$ the peak values are approximately twice the plateau value. For higher negative voltages the peak value increases fast while the plateau value only increases slowly. The DCMS values coincide quite well with the plateau where the measurements overlap $(520-600 \mathrm{~V})$.

The variation of the current pulse shape with chamber gas pressure is illustrated in Figure 4. The initial current peak becomes higher, and it takes longer time before the plateau develops, with higher gas pressures. For lower gas pressures a delay in plasma ignition can be observed (as has also been reported in an earlier work [9]). For all pressures, but the lowest, a second peak can be observed around 150-250 $\mu$ s. The peak current and plateau current values are plotted in Figure 5 for the studied chamber pressures. One can here observe that the current peak value saturate for high pressures at a value of $25 \mathrm{~A}$.

Finally, the current characteristics when varying the pulse off-time were investigated. The results can be seen in Figure 6. The way the measurements were done gives rise to an artifact, 
D. Lundin et al. Transition from a highly ionized plasma to a low density plasma in a high power impulse magnetron discharge

resulting in a shift of the current to lower values with decreasing off-time. (The current clamp only measures the $\mathrm{AC}$ component of the current, so that an offset compensating the DC component appears on the measured waveform.) It is here assumed that the final plateau current is the same for all measurements. This is motivated by the fact that the applied voltage and initial gas density stays the same resulting in an equal equilibrium current for different off-times. Correcting for this artifact still yields an effective decrease of the current peak with decreasing off-time.

\section{Discussion}

From the results presented above it is clear that by applying a pulsed voltage to the magnetron one achieves an initial high current that after a maximum around $\sim 40-50 \mu$ s starts to decrease and eventually reaches a steady-state level equivalent to the current level measured under the same experimental conditions when applying a constant voltage. We will here discuss what mechanisms determine the shape of the initial current peak and the transition to the steady-state plateau, beginning with the gas depletion and refill processes close to the target.

Gas heating and expansion. The increase in gas temperature and corresponding decrease in gas density in front of the target during a DCMS discharge, also known as gas rarefaction, has been extensively investigated during the last three decades both experimentally and theoretically $[10,11,12,13,14]$. The gas depletion in these models is assumed to be mainly through heating and corresponding thermal expansion, where the heating is due to collisions between the background gas and sputter-ejected target atoms as well as reflected sputtering gas atoms. A first Monte Carlo simulation of the neutral particle flow in HiPIMS was made by Kadlec [15], where it was shown that HiPIMS results in larger gas depletion than DCMS. With $I_{D}=1 \mathrm{kA}$ and $p=0.2$ $\mathrm{Pa}$, the neutral gas temperature was found to increase to $1 \mathrm{eV}$ after $50 \mu \mathrm{s}$, at which time the neutral gas density had decreased by $60-70 \%$ in a region extending $0.02 \mathrm{~m}$ above the racetrack. 
D. Lundin et al. Transition from a highly ionized plasma to a low density plasma in a high power impulse magnetron discharge

Direct current losses. During the high-current transient, a large fraction of the background gas, close to the cathode, can be lost through ionization and acceleration towards the target surface. This is a gas loss mechanism that becomes important for high currents, as in the case of HiPIMS, and seems not to have been accounted for in earlier publications cited above. To what extent this direct current loss will contribute to the total gas depletion depends on what will happen with the gas atom after impact on the target. This will be the subject for future studies, but here we can estimate the upper level of the initial loss of atoms through ionization and acceleration towards the target. For this purpose the beginning of a pulse is considered, where self-sputtering can be neglected. The full discharge current is then carried by Ar ions that arrive at the target and by emitted secondary electrons. Assuming that all the neutralized, returning, $\mathrm{Ar}$ atoms are energetic enough to pass quickly through the near-cathode region, the discharge current $I_{D}(t)$ corresponds to a reduction of the number of Ar atoms, $N_{A r}$, above the target, of $d N_{A r} / d t=I_{D}(1-\gamma) / e$, where $\gamma$ is the coefficient for secondary electron emission. The initial number of Ar atoms in the ionization volume above the target is $N_{A r, 0}=n_{A r, 0} V$. We can thus estimate the fractional loss of gas through direct current losses as

$$
\frac{\Delta n_{A r}}{n_{A r, 0}} \approx \frac{1}{n_{A r, 0} V} \int_{0}^{t} \frac{I_{D}(1-\gamma)}{e} d t .
$$

For a numerical approximation we take $\gamma=0.1$, and assume the gas loss to come from a region extending $2 \mathrm{~cm}$ above the racetrack. This gives a volume of $V \approx 1.1 \times 10^{-4} \mathrm{~m}^{3}$. With $I_{D}(t)$ from Figure 1 and $p=0.4 \mathrm{~Pa}\left(n_{A r, 0}=10^{20} \mathrm{~m}^{-3}\right)$, Eq. (1) gives about $40 \%$ gas loss after the highcurrent pulse.

Neutral gas refill. The gas loss mechanisms close to the target are counteracted by gas refill. For reference, refill with the thermal speed of Ar at room temperature, and over $0.02 \mathrm{~m}$, has a time constant of 50-100 $\mu$ s. The variation of peak current with off-time in Figure 6 is consistent with this estimate. For off-times as long as $3 \mathrm{~ms}$ we can assume the refill to be rather complete. 
D. Lundin et al. Transition from a highly ionized plasma to a low density plasma in a high power impulse magnetron discharge

The peak current is reduced below this " 3 ms value" by $50 \%$ for an off-time of $100 \mu$ s, indicating halfway refill of the gas. Notice that a much longer off-time is needed for complete refill: even with $750 \mu$ s off-time, the peak current is still $30 \%$ below the "3 ms value".

Bulk plasma processes. It has recently been found that the cross-conductivity, governing the transport of charged species, is much higher for HiPIMS $[16,17,18,19]$ than for DCMS [20, 21]. It is therefore expected that there is a transition from an anomalously high HiPIMS crossconductivity to a lower DCMS Bohm conductivity as the current approaches the plateau-level in the pulse. This change mainly influences the rather weak electric field in the bulk plasma [22]. However, we cannot exclude that it has an important effect on the observed transition.

Another aspect affecting the shape of the current decrease after the peak (phase II in Figure 1) is a plasma pressure wave. It is generated by the dense and hot plasma during each voltage pulse and expands with approximately the ion acoustic speed $\left(\sim 10^{3} \mathrm{~m} / \mathrm{s}\right)[9,16]$. Furthermore, the simulations by Kadlek [15] showed a neutral gas shock-like wave with a speed of $\leq 10^{3} \mathrm{~m} / \mathrm{s}$, moving away from the target. As demonstrated by Alami et al. [9] these types of pressure waves can return to the cathode region after interacting with the chamber wall and affect the behavior of the discharge. The characteristic size of our system is about $0.2 \mathrm{~m}$, which would yield an effect at the cathode after $\sim 400 \mu$ s. We have not been able to confirm such an effect in the present case, but cannot exclude that the structure of the decreasing current around 150-250 $\mu$ s for higher pressures (Figure 4) is due to this phenomenon.

In summary we envisage the following time development. Due to gas heating and current losses close to the target, there is an initial directed outflow of gas. When the gas density decreases the ionization rate per electron, which is proportional to the neutral density, also decreases. At some point a maximum ionization rate is reached, and this defines the reversal point between region I and II in Figure 1. When the current decreases, the plasma density eventually begins to drop due to the decreased amount of secondary electrons ejected from the target. As a result both the reduced current losses and the decreased collisional heating cause the gas 
D. Lundin et al. Transition from a highly ionized plasma to a low density plasma in a high power impulse magnetron discharge

depletion to proceed more slowly, until equilibrium with the gas inflow from the surrounding volume is reached. The shape of the high-current pulse varies when varying the Ar pressure, as seen in Figure 4. The decay time measured from the peak target current to the beginning of the plateau has been plotted in Figure 7. The longer decay time for higher pressures might be due to a combination of two effects. First, the relative density depletion through current losses from Eq. (1), during the first $100 \mu \mathrm{s}$, is smaller for increased gas pressure. Second, there is a lower diffusion constant for higher gas pressures, and also a higher thermal load of a denser gas.

It should be mentioned that recent studies of the discharge current characteristics by Anders et al. $[23,24]$ have shown that some materials (such as Ti) in combination with high negative discharge voltages can lead to that the plateau current starts to increase beyond the value of the initial peak current. This has been proposed to be due to a mode of sustained self-sputtering and is related to the appearance of multiply charged ions. In the case of $\mathrm{Cr}$, which is used in this work, there are no reports of such behavior.

From the discussion above it is suggested that gas depletion leads to lower currents, for a given voltage, both in long HiPIMS pulses and in DCMS discharges. Short $(<100 \mu s)$ HiPIMS pulses are not in the density and heat equilibrium found in DCMS plasmas, but with increasing pulse lengths they are effectively moving towards a DCMS-like regime. The current plateau can thus be explained by the pulse being long enough for the plasma to reach equilibrium with regard to several interacting mechanisms: neutral gas heating, current losses, refill, and possibly also plasma and gas pressure waves. The fact that the experimentally measured HiPIMS plateau current coincides with the DCMS values for the same applied voltage can be understood, since we now are in the same equilibrium regime. Consequently, this leads to the notion that the very high currents in short HiPIMS pulses are possible because they fall in a transition state before severe gas depletion starts to dominate the current behavior. Corresponding initial current peaks, before gas depletion, are possible also in the case of an applied discharge voltage less than the normal HiPIMS values. The fact that they are usually not observed during ignition of ordinary 
D. Lundin et al. Transition from a highly ionized plasma to a low density plasma in a high power impulse magnetron discharge

DCMS plasmas can be explained by the design of the DCMS power supplies. Conventional power supplies have a current limit of typically a few Amperes. In addition, the voltage is often ramped up to avoid a current overshoot.

\section{Conclusions}

Discharge pulses longer than the typical HiPIMS pulse length have been applied to a conventional magnetron sputtering system. From the current characteristics it was found that an initial high-current peak was followed by a stable plateau at lower current, which lasted until the end of the pulse. The current behavior was found to be strongly correlated with the chamber gas pressure, where increasing gas pressure resulted in increasing peak current and plateau current. Furthermore, the current plateau values coincide with measured current values for DCMS using the same applied voltage. Based on experimental results and estimates of plasma transport and gas dynamics during the discharge, it is suggested that high HiPIMS currents are usually possible only when the pulses are so short that depletion of the sputtering gas is avoided. Longer pulses ultimately lead to a transition from a high voltage, high current HiPIMS regime to a DCMS-like high voltage regime with lower current.

\section{Acknowledgements}

This work was financially supported by the European Commission within the $6^{\text {th }}$ framework (integrated project: InnovaTiAl), the Swedish Research Council, and the Swedish Foundation for Strategic Research. 
D. Lundin et al. Transition from a highly ionized plasma to a low density plasma in a high power impulse magnetron discharge

\section{Figures}

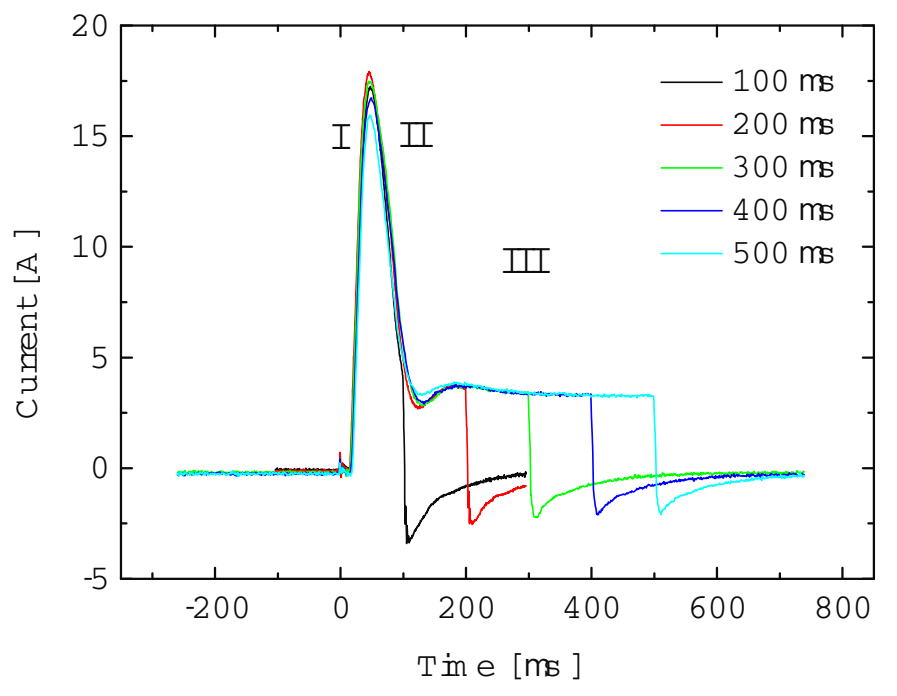

Figure 1. Current characteristics plotted for different discharge pulse lengths. The low current plateau is stable at about $3.5 \mathrm{~A}$, but gets cut off for different pulse lengths using the same U-I values and off-times. The negative discharge potential was $700 \mathrm{~V}$ at an Ar pressure of $0.4 \mathrm{~Pa}$. The different discharge regimes are marked I-III.

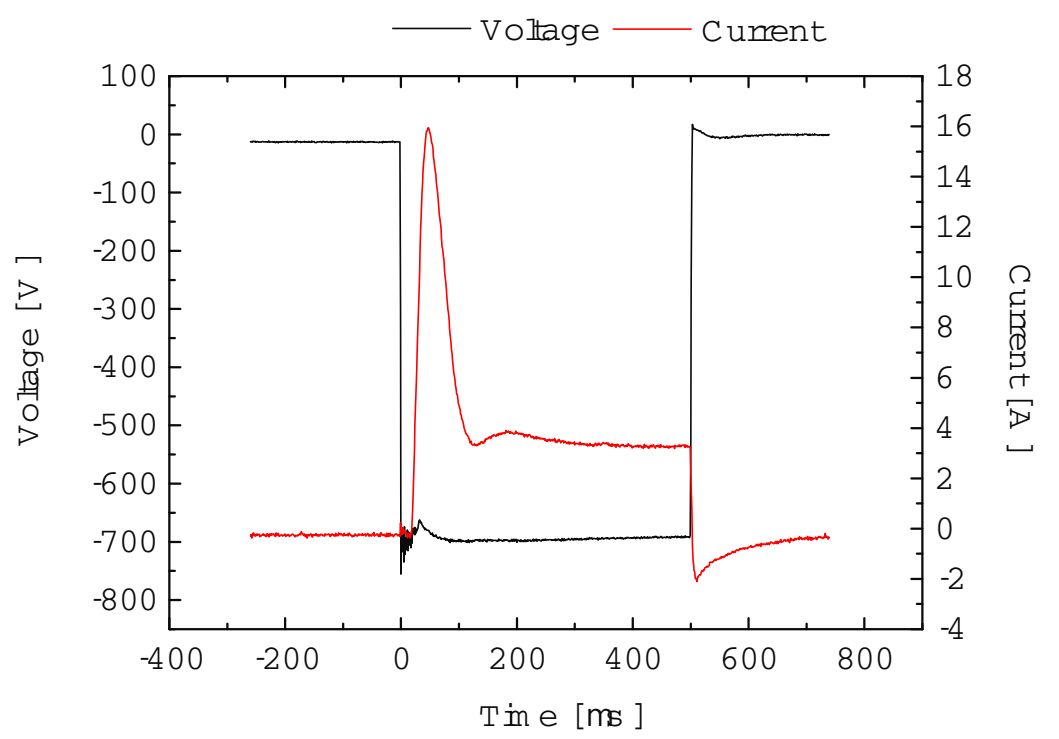

Figure 2. Discharge voltage and current characteristics in a $500 \mu$ s pulse. The negative discharge potential was $700 \mathrm{~V}$ at an $\mathrm{Ar}$ pressure of $0.4 \mathrm{~Pa}$. 
D. Lundin et al. Transition from a highly ionized plasma to a low density plasma in a high power impulse magnetron discharge

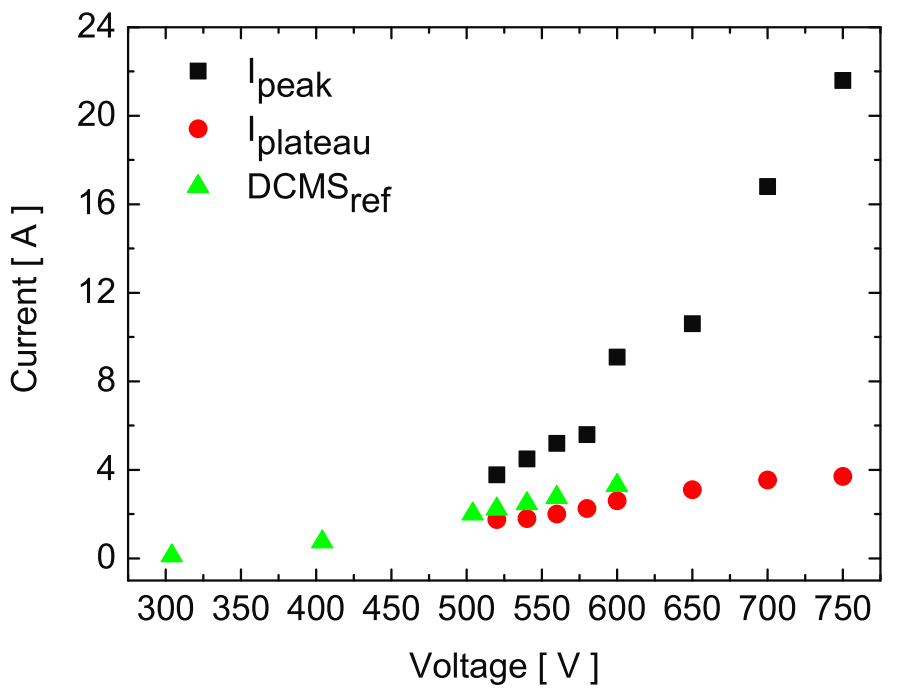

Figure 3. The initial high-current peak and the current plateau plotted for different applied negative voltages. A reference current for the DCMS case is also plotted. The current plateau values coincide well with the DCMS values for the same applied voltage. The measurements were performed at an Ar pressure of $0.4 \mathrm{~Pa}$, using $500 \mu \mathrm{s}$ on-time and $9.9 \mathrm{~ms}$ off-time.

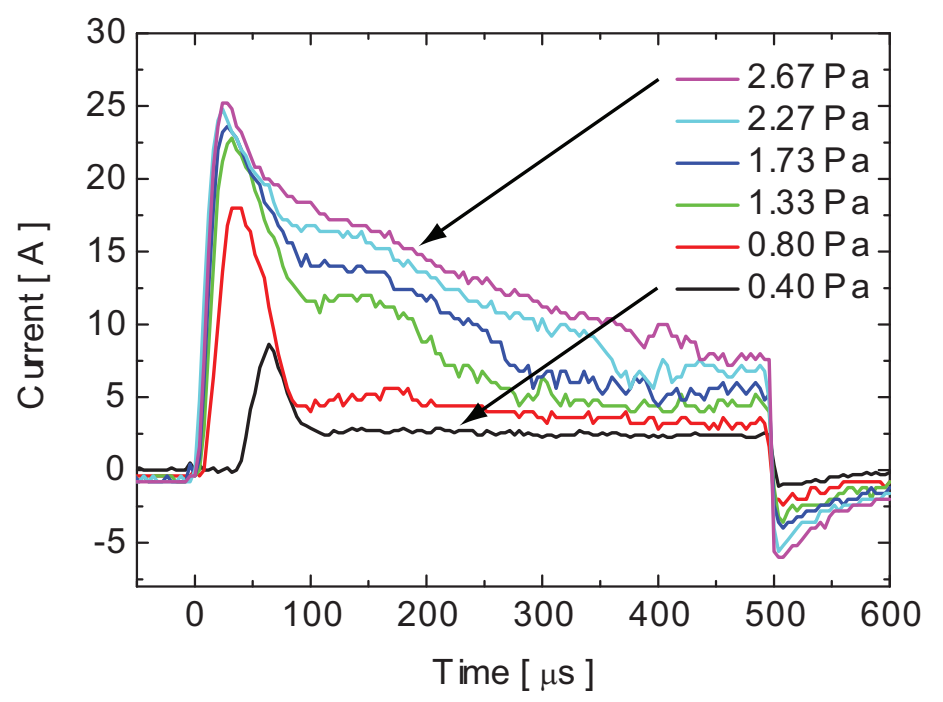

Figure 4. Current curves plotted for different Ar pressures. The current plateau appears later in the pulse when the pressure is increased and the initial high-current peak is consequently broadened. The negative discharge potential was $650 \mathrm{~V}$ using $500 \mu \mathrm{s}$ on-time and $9.9 \mathrm{~ms}$ off-time pulses. 
D. Lundin et al. Transition from a highly ionized plasma to a low density plasma in a high power impulse magnetron discharge

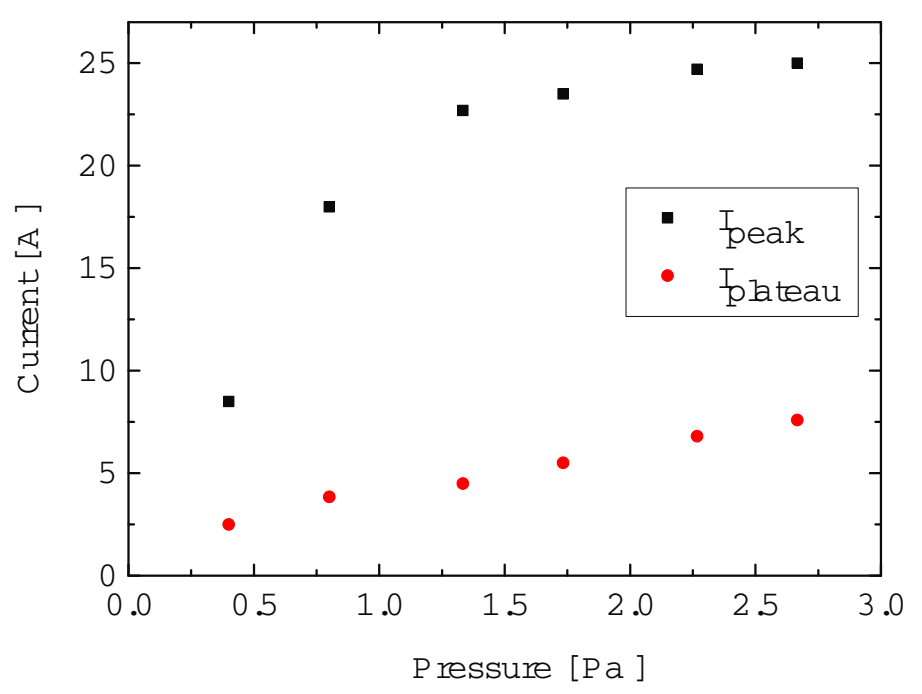

Figure 5. The peak current and the plateau current plotted versus Ar gas pressure. The peak current saturates at pressures above $\sim 1.5 \mathrm{~Pa}$, while the plateau current shows a close to linear increase in the same range. The data is taken from Figure 4. 
D. Lundin et al. Transition from a highly ionized plasma to a low density plasma in a high power impulse magnetron discharge

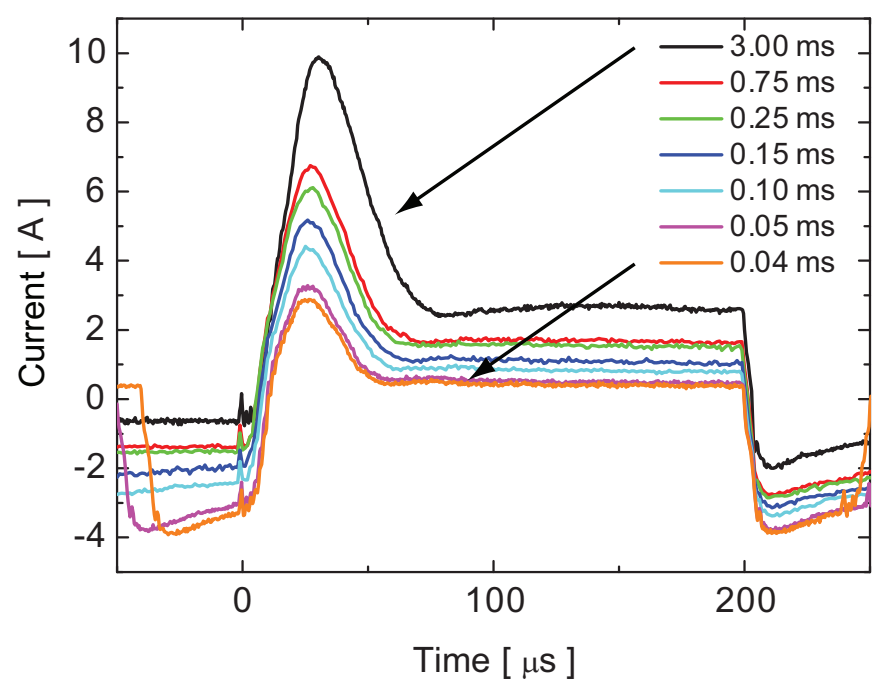

Figure 6. Current characteristics plotted for different pulse off-times. Decreasing off-times result in lower peak current. The measurements were performed at an Ar pressure of $0.4 \mathrm{~Pa}$, using a negative discharge potential of $650 \mathrm{~V}$ with $200 \mu$ s on-time.

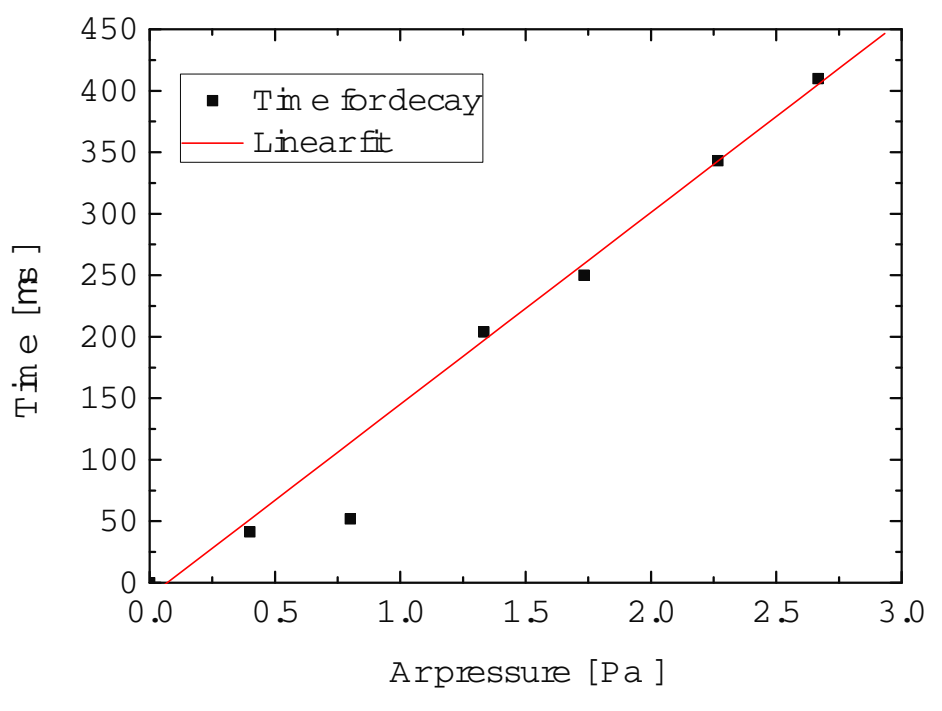

Figure 7. Time for current decay from current peak to current plateau plotted against varying Ar pressure. The data is taken from Figure 4. 
D. Lundin et al. Transition from a highly ionized plasma to a low density plasma in a high power impulse magnetron discharge

\section{References}

[1] Mozgrin D V, Fetisov I K and Khodachenko G V 1995 Plasma Phys. Rep. 21400

[2] Kouznetsov V, Macák K, Schneider J M, Helmersson U and Petrov I 1999 Surf. Coat. Technol. 122 290

[3] Macák K, Kouznetsov V, Schneider J M, Helmersson U and Petrov I 2000 J. Vac. Sci. Technol. A 18 1533

[4] Gudmundsson J T, Alami J and Helmersson U 2002 Surf. Coat. Technol. 161249

[5] Alami J, Persson P O Å, Music D, Gudmundsson J T, Bohlmark J and Helmersson U 2005 J. Vac. Sci. Technol. A 23278

[6] Wallin E, Selinder T I, Elfwing M and Helmersson U 2008 Europhys. Lett. 8236002

[7] Konstantinidis S, Dauchot J P, Ganciu M and Hecq M 2006 J. Appl. Phys. 99013307

[8] Alami J, Sarakinos K, Mark G and Wuttig M 2006 Appl. Phys. Lett. 89154104

[9] Alami J, Gudmundsson J T, Bohlmark J, Birch J and Helmersson U 2005 Plasma Sources Sci. Technol. 14525

[10] Hoffman D W 1985 J. Vac. Sci. Technol. A 3561

[11] Rossnagel S M 1988 J. Vac. Sci. Technol. A 619

[12] Palmero A, Rudolph H and Habraken F H P M 2005 Appl. Phys. Lett. 87071501

[13] Palmero A, Rudolph H and Habraken F H P M 2006 Thin Solid Films 515631

[14] Kolev I and Bogaerts A 2008 J. Appl. Phys. 104093301

[15] Kadlec S 2007 Plasma Proc. Polym. 4 S419

[16] Bohlmark J, Helmersson U, VanZeeland M, Axnäs I, Alami J and Brenning N 2004 Plasma Sources Sci. Technol. 13654

[17] Lundin D, Helmersson U, Kirkpatrick S, Rohde S and Brenning N 2008 Plasma Sources Sci. Technol. 17025007

[18] Lundin D, Larsson P, Wallin E, Lattemann M, Brenning N and Helmersson U 2008 Plasma Sources Sci. Technol. 17035021

[19] Vetushka A and Bradley J W 2007 J. Phys. D 402037 
D. Lundin et al. Transition from a highly ionized plasma to a low density plasma in a high power impulse magnetron discharge

[20] Rossnagel S M and Kaufman H R 1987 J. Vac. Sci. Technol. A 52276

[21] Bradley J W, Thompson S and Aranda Gonzalvo Y 2001 Plasma Sources Sci. Technol. 10490

[22] Brenning N, Axnäs I, Raadu M A, Lundin D and Helmerson U 2008 Plasma Sources Sci. Technol. 17 045009

[23] Anders A, Andersson J and Ehiasarian A 2007 J. Appl. Phys. 102113303

[24] Anders A 2008 Appl. Phys. Lett. 92201501 